\title{
Management of Stiffness following Total Knee Arthroplasty: International Survey on Surgeon Preferences
}

\author{
Karadi Hari Sunil Kumar ${ }^{1, *}$, Georgios Mamarelis ${ }^{2}$, Matthew Pettit ${ }^{3}$, and Vikas Khanduja ${ }^{4}$ \\ 1 Specialty Registrar in Trauma \& Orthopaedics, Addenbrooke's Hospital, Hills Road, Cambridge CB2 0QQ, UK \\ 2 Specialty Registrar in Trauma \& Orthopaedics, Royal London Hospital, Whitechapel, London E1 1BB, UK \\ 3 Medical Student, University of Cambridge, CB2 8PQ, UK \\ ${ }^{4}$ Consultant Orthopaedic Surgeon, Addenbrooke's Cambridge University Hospital, Hills Road, Cambridge CB2 0QQ, UK
}

Received 15 December 2020, Accepted 10 February 2021, Published online 30 April 2021

\begin{abstract}
Introduction: Stiffness following total knee arthroplasty (TKA) is a challenging complication and can result in a poor functional outcome. There is considerable debate concerning the definition, work-up, and optimal management of this complication. The aim of this study was to record the definition of stiffness, management practices, and expectations of outcome among surgeons from an international community using a peer-reviewed questionnaire. Methods: A 23-item peer-reviewed online questionnaire was sent to all members of SICOT to gauge and record the management practices and expectations of outcome in the management of patients with stiffness following TKA. Results: A total of 315 surgeons completed this peer-reviewed questionnaire. Manipulation under anaesthesia (MUA) was the preferred treatment option for stiffness post-TKA, with a majority of the surgeons opting to carry out this procedure between 6 and 12 weeks following the index TKA. Physiotherapy and a continuous passive motion device were also used by the majority of surgeons following MUA, as additional treatment measures. Discussion: MUA is perceived to be a safe and effective primary treatment option for stiffness following TKA. It is best performed between weeks 6 and 12 with expected gains in range of motion from 10 to 20 degrees in $75 \%$ of patients.
\end{abstract}

Key words: Total knee arthroplasty, Postoperative stiffness, Manipulation under anesthesia, Management, Outcomes.

\section{Introduction}

Total knee arthroplasty (TKA) is being performed increasingly for treating end-stage arthritis of the knee [1]. The lifetime risk of undergoing a primary TKA for a population older than 25 years is $7 \%$ for males and $9.5 \%$ for females [1]. The goal of performing a TKA is to achieve a painless and stable knee joint with a functional range of motion (ROM) [2]. Patient satisfaction following TKA is influenced by a multitude of factors of which post-operative improvement in range of movement and meeting pre-operative patient expectation are key to achieving successful outcomes [3]. The 10-year implant survival rate is reported to be $96.1 \%$, and the 20 -year implant survival rate is $89.7 \%$ [4]. However, about $15-20 \%$ of the patients remain dissatisfied following the procedure and one of the reasons for this dissatisfaction is poor post-operative ROM [4]. A ROM of $10^{\circ}-95^{\circ}$ is required for walking and climbing stairs. Some activities of daily living require the knee joint to flex up to $115^{\circ}$, for example tying shoelaces while sitting [5], and some activities like sitting cross-legged and kneeling while praying require flexion beyond.

\footnotetext{
*Corresponding author: drkhskumar@yahoo.com
}

Post-operative stiffness is defined as an inadequate ROM causing functional limitations in activities of daily living and occurs in approximately 5-6\% of TKAs. And some studies report an incidence of up to $20 \%$ [6-9]. However, caution is needed when interpreting these publications as ROM based definitions of stiffness used in the literature are inconsistent $[6,10,11]$.

Stiffness following TKA can occur as a result of several conditions (Table 1) with different treatment options (Table 1). Arthrofibrosis is the primary cause of stiffness [2] and manipulation under anaesthesia (MUA) has been described as the first line of intervention from as early as 2 weeks to 4 months postoperatively [9]. However, there is no consensus for the optimal time to perform an MUA, whether MUA should actually be the first line of intervention and debate continues on post-MUA management of this difficult cohort of patients. Our group is keen on understanding these differences and has published on the subject previously [2, 12].

Currently, there is no clear definition of stiffness following TKA and a lack of a clear management algorithm, especially with the timing of MUA for stiffness. It is vital to understand the current management practices and expectations of outcomes 
Table 1. Causes of stiffness following TKA and treatment options.

\begin{tabular}{ll}
$\begin{array}{l}\text { Causes of stiffness post- } \\
\text { TKA }\end{array}$ & \multicolumn{1}{c}{ Treatment options available } \\
\hline $\begin{array}{l}\text { Arthrofibrosis } \\
\text { Infection }\end{array}$ & $\begin{array}{l}\text { Physiotherapy } \\
\text { Continuous passive motion (CPM) } \\
\text { device }\end{array}$ \\
$\begin{array}{l}\text { Implant malposition } \\
\text { Dislocation of TKA } \\
\text { Fracture of polyethylene } \\
\text { Peri-prosthetic fracture }\end{array}$ & $\begin{array}{l}\text { Arthroscopic arthrolysis } \\
\text { Open arthrolysis }\end{array}$ \\
\hline
\end{tabular}

concerning the management of stiffness following TKA across the international community. This would help surgeons have a better understanding of the management of this important problem.

The aim of the study was therefore to (1) define stiffness following TKA and work-up for this problem, (2) analyse the timing of MUA for stiffness following TKA, (3) identify the different modalities and duration of rehabilitation used following MUA, (4) assess the improvement in ROM after MUA and recurrence of stiffness, (5) ascertain complications of MUA experienced by surgeons, and (6) different patient-reported outcome measures (PROMs) used and patient satisfaction.

\section{Methods}

A pilot survey was prepared following an in-house discussion of the research team addressing the study aims. This contained sections devoted to the surgeon's current practice, including the diagnosis and investigation of stiffness, management options, rehabilitation protocol, and follow-up schedule. The questions were of a multiple-choice format, and the participants were encouraged to provide comments where necessary. The pilot survey was sent to an independent group of 20 senior knee arthroplasty surgeons in the UK, USA, Canada, and Australia. This process filtered ambiguities and the surgeons commented on the transparency and comprehensiveness of the questions, providing suggestions to improve the quality of the survey. The questionnaire was modified following the pilot survey and a final 23-item questionnaire-based survey (Supplementary Table 1s) was constructed and delivered through an online medium using Survey Monkey (Survey Monkey®, San Mateo, California Office, One Curiosity Way, San Mateo, CA 94403).

We chose SICOT as a target organization to undertake the survey because of its international profile with global representation. SICOT is an International Orthopaedic Organisation, governed by Belgian Law, with surgeon members from 110 member countries [13]. The online survey was delivered via e-mail to all SICOT members, which at the time of the survey were approximately 2500 . A reminder email was sent to all SICOT members at 8 and 16 weeks following the initial email to improve the response rate. All data were stored anonymously on the survey monkey website with the facility to export the information to perform descriptive statistical analysis. This study received approval from the Institution's Audit Department.

\section{Results}

We received a response from 315 members of the organization after two reminders and that reflects the number of surgeons potentially interested in knee arthroplasty in the membership.

\section{Geographical location and experience of those completing the survey}

The responses received were from 26 countries across 6 continents. The majority of the responses were from Asia (43.2\%) and Europe (35.6\%). In addition, we received responses from Africa, North America, Australia, and South America (Figure 1). Forty percent of the responding surgeons were working in a teaching hospital. There was a wide range of experience in the respondents ranging from orthopaedic trainees to those with more than 25 years of independent practice (Figure 2). More than 55\% of the participants performed 50 or more TKAs each year (Figure 3 ).

\section{Definition of TKA stiffness and work-up}

Considerable variability was found among the participating surgeons in how they defined stiffness following TKA (Figure 4). There was no regional variability in the definition of stiffness. The majority of the participants $(66.7 \%)$ perceived that less than $3 \%$ of patients developed a stiff knee post-operatively, while $19.5 \%$ felt that 3-5\% developed stiffness following TKA. A majority (76.3\%) also performed blood tests, including C-reactive protein (CRP) and Erythrocyte Sedimentation Rate (ESR), as a part of the investigations for stiffness etiology. In addition, $89.9 \%$ of the participants performed plain radiographs, $44.4 \%$ performed a Computed Tomography (CT) scan, and $14.9 \%$ performed Single Photon Emission Computed Tomography (SPECT) of the knee joint to rule out implant malposition and infection respectively.

\section{Timing of MUA}

The majority of participating surgeons (90.2\%) performed MUA for arthrofibrosis. With regards to the timing of MUA $54.6 \%$ considered that MUA for stiffness should be performed between 6 and 12 weeks following the index TKA, whereas $20.1 \%$ would perform MUA within the first 6 weeks, $17.4 \%$ between 12 and 20 weeks, and $7.8 \%$ would consider MUA after more than 20 weeks (Figure 5). A limited number $(9.8 \%$ ) would not perform MUA for stiffness following TKA.

\section{Rehabilitation following MUA}

The majority $(95.5 \%)$ of the participating surgeons offered physiotherapy to their patients following MUA; $60.7 \%$ of the surgeons offered physiotherapy routinely for 6 weeks and $28.2 \%$ for up to 3 months. Additionally, $71.7 \%$ of the participants used a Continuous Passive Motion (CPM) device, with 


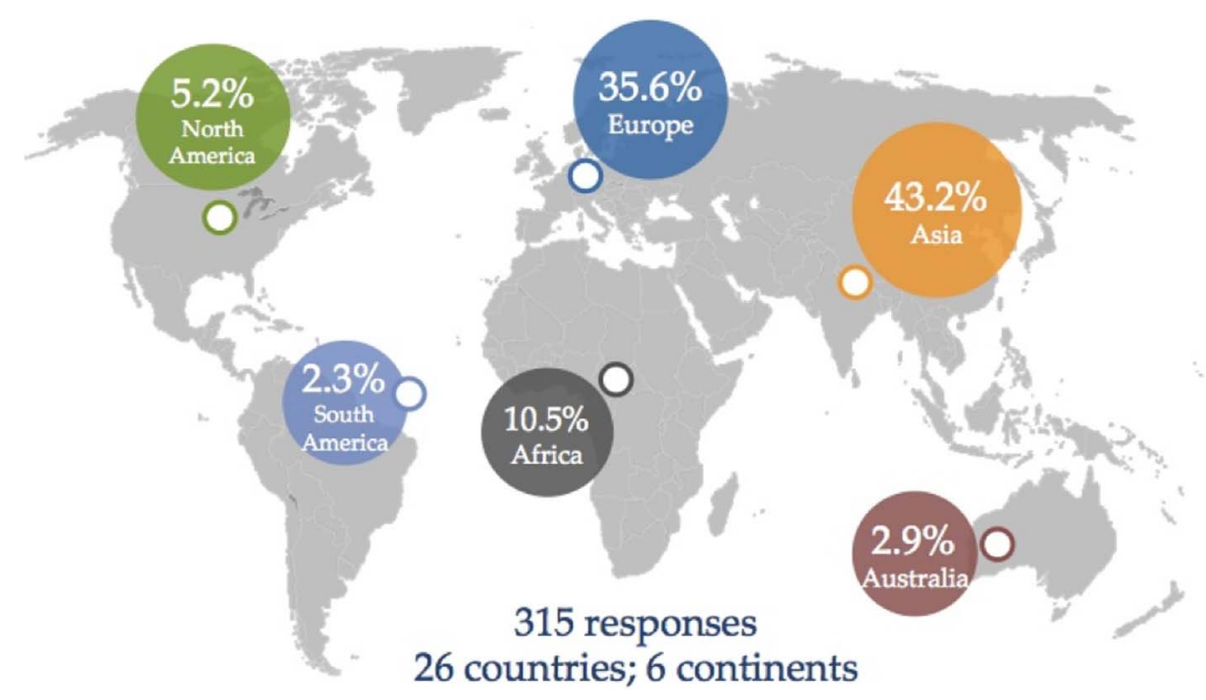

Figure 1. Survey participants by continent.

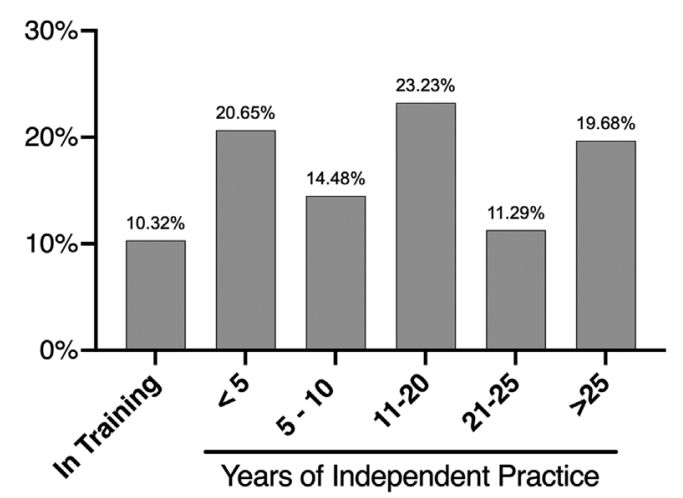

Figure 2. Results of the survey question: How many years have you been in independent practice?

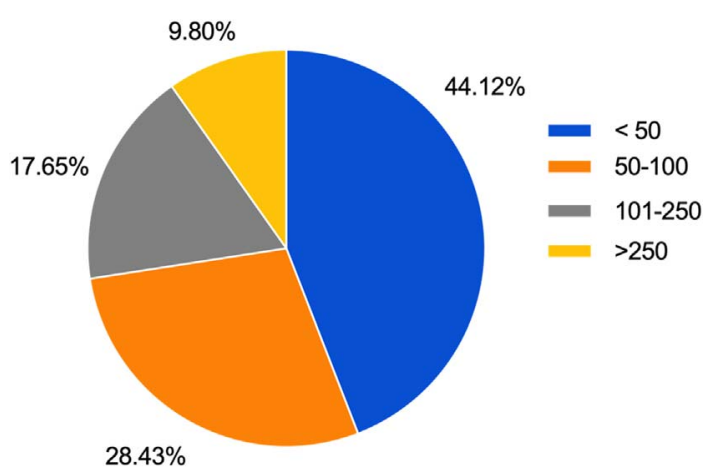

Figure 3. Results of the survey question: How many Total Knee Arthroplasties do you perform annually?

a varied duration of use. CPM was used by $38.8 \%$ for inpatients until the achievement of the target ROM, $16.9 \%$ of the patients were discharged home with CPM if necessary, to achieve target ROM and $15.8 \%$ used CPM only for inpatients irrespective of achievement of ROM.

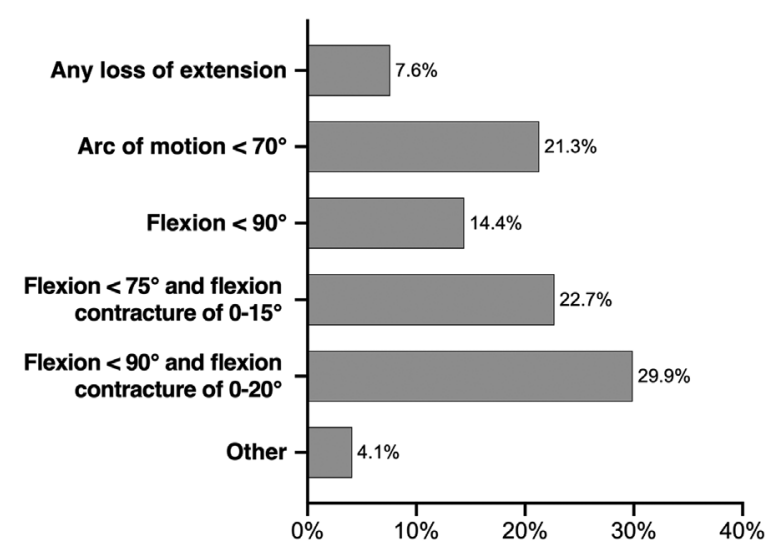

Figure 4. Results of the survey question: How do you define stiffness following Total Knee Arthroplasty?

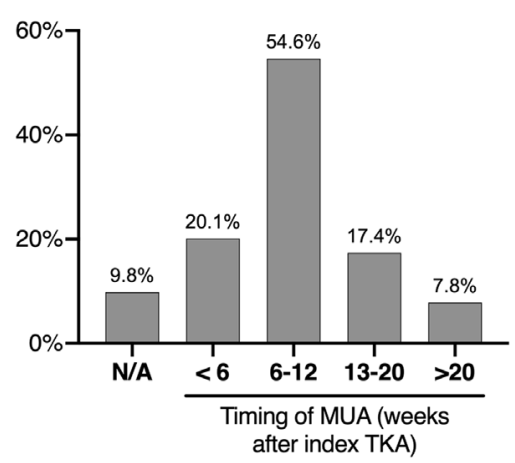

Figure 5. Results of the survey question: How long after the index TKA would you perform MUA? N/A indicates surgeons who would choose not to perform MUA for stiffness following TKA.

\section{Improvement in the ROM, recurrence rates, and treatment options}

The participating surgeons who performed MUA for stiffness following TKA expected improvement in ROM, 
almost $75 \%$ of participants expected an improvement of a minimum of $10^{\circ}-20^{\circ}$. A further sub-group analysis revealed that $31 \%$ of participants expected between $10^{\circ}$ and $20^{\circ}$ gain in the ROM, compared to $41 \%$ expecting $20^{\circ}-30^{\circ}, 14.8 \%$ expecting $30^{\circ}-40^{\circ}$ and $9.5 \%$ expecting $>40^{\circ}$ improvement in ROM following MUA.

The perceived recurrence rate of stiffness following MUA varied among participating surgeons: $32 \%$ estimated that the recurrence rate was less than $1 \%, 26 \%$ estimated that it was $1-5 \%, 18.6 \%$ estimated that it was $5-10 \%$, and $23.3 \%$ estimated recurrence in more than $10 \%$ of cases.

In patients with recurrence of stiffness following MUA, nearly half $(46.4 \%)$ of the participating surgeons elected not to perform a second MUA. Only $24.4 \%$ occasionally performed a second MUA and $17.7 \%$ would only perform a second MUA at the patient's request. Thirty seven percent of the participants elected to combine MUA with arthroscopic arthrolysis, while $21.3 \%$ of participants would proceed to open arthrolysis and $12.9 \%$ would consider revision surgery if necessary. Additionally, $12.6 \%$ of the participants considered physiotherapy alone as appropriate management in case of a recurrence of stiffness.

\section{Complications following MUA}

Manipulation under anaesthesia for stiffness following TKA was considered a safe procedure by the majority of participating surgeons, $71.9 \%$ estimated that the rate of complications including fracture, patella tendon rupture, and algodystrophy was less than $1 \%$. The remainder of the participants estimated that their complication rates were $1-3 \%(19.4 \%)$ or more than $3 \%(8.7 \%)$.

\section{PROMs and patient satisfaction}

A variety of scoring systems were used to assess TKA outcomes among the participating surgeons. The most popular scoring system was the Knee Society System (61.9\%), followed by the Oxford Knee Score (28.6\%). The majority of participants $(60 \%)$ estimated that their patients are either satisfied or very satisfied with the outcome of MUA for stiffness following TKA, 32.5\% estimated their patients to be neutral with regard to MUA outcome and $7.5 \%$ estimated that the patients would be dissatisfied with the outcome.

\section{Discussion}

This is the first international survey that investigates the practices of orthopaedic surgeons concerning the diagnosis, investigation, and management of stiffness following TKA. We received responses from 315 orthopaedic surgeons, with varying experience, ranging from trainees to those with over 25 years of independent orthopaedic practice. The results revealed a wide variation in practice. Although $44.1 \%$ of the survey respondents performed less than 50 TKAs per year, the majority (55.9\%) performed more than 50 TKAs per year including $9.8 \%$ who performed more than 250 TKAs per year (Figure 3). The participation of surgeons with a keen interest in TKA helped to improve the validity of these results.
We accept some limitations of our study. Firstly, electronic surveys are prone to a low response rate [14]. We chose this medium as this provided worldwide access and also afforded a quick response with a follow-up email reminder to complete the same. Our response rate was low with just over $12.6 \%$ of the total SICOT membership responding, although this still afforded sufficient numbers for data analysis and reflects the number of surgeons interested in knee arthroplasty in a generalist orthopaedic society. Secondly, the majority of the participants were from Asia and Europe (Figure 1), this may indirectly reflect the represent SICOT membership from these regions. Increased participation elsewhere globally may have revealed regional variations in practice if any. Thirdly, even though the questionnaire was comprehensive with 23 -items, we were not able to capture all possible data due to the inherent limitation of survey-based research.

In this survey there was a wide variation in how participants defined stiffness following TKA, reflecting the breadth of definitions in the current literature [10]. In the future, it is recommended that the international consensus on the definition of stiffness achieved by The Knee Joint Fibrosis Working Group should be adopted which states a requirement for "a restricted ROM in flexion or extension, or both flexion and extension" in order to diagnose post-operative arthrofibrosis [11]. Additionally, the group has precisely graded the severity of arthrofibrosis into mild, moderate, and severe depending on either the restriction of extension $\left(5^{\circ}-10^{\circ}, 11^{\circ}-20^{\circ},>20^{\circ}\right)$ or the flexion range $\left(90^{\circ}-100^{\circ}, 70^{\circ}-89^{\circ},<70^{\circ}\right)$ [11]. The proportion of patients estimated to undergo MUA for stiffness following TKA ranges from $2.2 \%$ to $6 \%$ [15]. Our survey reinforced this with $86.3 \%$ of participating surgeons estimating that less than $5 \%$ of patients developed stiffness following TKA.

The optimal timing of MUA after TKA is controversial [19]. Some studies suggest that MUA within 12 weeks from TKA achieves better ROM outcomes compared with MUA performed after longer intervals $[15,16]$ one such study suggests that the therapeutic benefit is lost 26 weeks after TKA [16]. Other studies have established no difference in outcome following MUA performed before or after 12 weeks [17]. In our survey, $54.5 \%$ of the participating surgeons advised MUA within 6-12 weeks of TKA and $20.1 \%$ advised that MUA should be performed within 6 weeks of TKA, indicating that current surgical practice is to prefer MUA at generally shorter intervals $(<12$ weeks) from the index TKA.

Yercan et al. proposed that thorough investigations be performed prior to MUA to rule out infection, algodystrophy, or surgical error which are contraindicated for MUA [6]. Additionally, the Knee Joint Fibrosis Working Group suggests that a clinical diagnosis of joint fibrosis may be made after excluding other causes of stiffness, using investigations including plain radiographs, CT scans, serology, and aspiration [15]. Our survey demonstrated a similar sentiment, the most common investigations performed were blood tests, plain radiographs, and CT scans, which were preferred by $76.3 \%, 89.9 \%$, and $44.6 \%$ of the participating surgeons respectively.

Issa et al. reported that the mean gain in flexion following MUA in their study was $33^{\circ}$, ranging from $5^{\circ}$ to $65^{\circ}$, with a gain of $36.5^{\circ}$ for early MUA compared to $17^{\circ}$ for late MUA [16]. Our survey showed $41 \%$ of participants expected between 


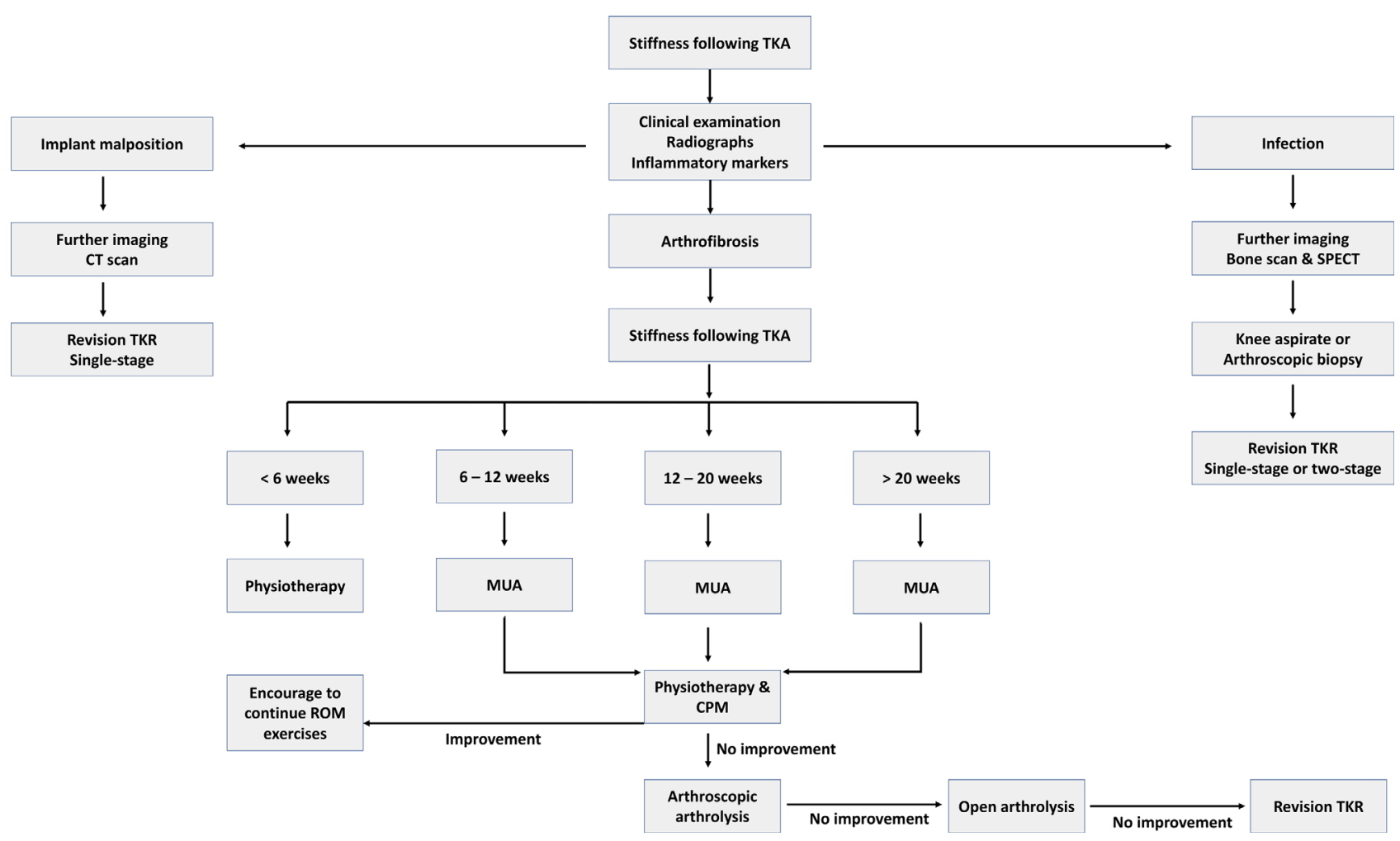

Figure 6. Algorithm for the treatment of stiffness post total knee arthroplasty.

Table 2. Surgical options for stiffness and their possible complications.

\begin{tabular}{|c|c|}
\hline Procedure & Complications \\
\hline \multirow[t]{6}{*}{ MUA } & Bleeding \\
\hline & Wound dehiscence \\
\hline & Peri-prosthetic fracture of femur or tibia \\
\hline & Dislocation of the TKR \\
\hline & Fracture of the tibial post of polyethylene \\
\hline & Rupture of patellar tendon \\
\hline \multirow[t]{4}{*}{ Arthroscopic arthrolysis } & Infection \\
\hline & Damage to bearing surface of TKR \\
\hline & Damage to polyethylene insert \\
\hline & Bleeding \\
\hline \multirow[t]{4}{*}{ Open arthrolysis } & Infection \\
\hline & Damage to bearing surface of TKR \\
\hline & Damage to polyethylene insert \\
\hline & Wound complication \\
\hline \multirow[t]{3}{*}{ Revision TKR } & Infection \\
\hline & Wound complication \\
\hline & Recurrence of stiffness \\
\hline
\end{tabular}

$10^{\circ}$ and $20^{\circ}$ gain in ROM, with the remaining participants expecting greater and lesser gains in roughly equal proportions. There is no consensus on the usefulness of repeated MUA [18].

Additional use of CPM in combination with physiotherapy has been shown to be beneficial during the early rehabilitation phase following a primary TKA [18, 19]. Although a study from Boese et al. reported no clinically significant difference in ROM with the postoperative use of CPM [20]. A recent national survey from the UK reported that $68 \%$ of surgeons would routinely use CPM post manipulation [21]. A total of $72 \%$ of our study participants used CPM following MUA but for a varying duration similar to the UK practice, denoting that the CPM usage was similar across the globe. Post-operative physiotherapy following TKA is a key factor for maintaining ROM and therefore satisfactory outcomes [5, 6, 19]. Our survey reinforced this, as $89 \%$ of participants using physiotherapy for at least 6 weeks following MUA, and 27\% using physiotherapy for 3 months.

There are four typical management options for stiffness following TKA. After MUA these are arthroscopic arthrolysis, open arthroscopic arthrolysis, and revision TKA [8, 10, 11]. Our survey showed that in recurrent stiffness following MUA, $36.8 \%$ of participants would perform MUA with arthroscopic arthrolysis and $21.3 \%$ would proceed directly to open arthrolysis. The Knee Joint Fibrosis Working Group has also suggested escalation along with these four treatment options [15].

Even though MUA is a non-invasive procedure, it is crucial to ensure that the benefits outweigh the risks and possible complications. MUA is perceived to be a safe procedure with $71.8 \%$ of participating surgeons estimating a complication rate of less than $1 \%$ following MUA. The complications for each of the procedure is listed in Table 2.

\section{Strengths of the study}

This is the first study to assess the management preferences of surgeons across the globe towards stiffness following TKA. The participating surgeons had different levels of experience both in terms of years in independent practice and the number 
of TKAs performed annually, which can be generalized to the whole orthopaedic community. In addition, the final questionnaire was devised following a preliminary validity assessment of the pilot survey by 20 senior knee arthroplasty surgeons from the International Community.

A timely accurate diagnosis of stiffness following total knee arthroplasty is essential to appropriately manage the condition and ensure good patient satisfaction. A simplified treatment algorithm is shown in Figure 6, which applies to the majority of the cases presenting with stiffness following TKA.

\section{Conclusions}

Stiffness is a well-recognized complication following TKA, which can result in a poor functional outcome. A thorough evaluation of patients with a stiff knee following TKA is essential to correctly diagnose arthrofibrosis as the cause of stiffness. MUA is widely recognized as the optimal first-line treatment to improve ROM in postoperative arthrofibrosis. Improvement in $\mathrm{ROM}$ of $10^{\circ}$ to $20^{\circ}$ can be expected in $75 \%$ of patients. Post-operative physiotherapy for $6-12$ weeks is believed to be useful to maintain the gain in ROM. In cases where there is a recurrence of stiffness following MUA, our survey suggests a second MUA with or without arthrolysis should be considered. MUA is perceived as a safe procedure with a low complication rate, where a good functional outcome can be expected.

\section{Supplementary Material}

The supplementary material of this article is available at https://www.sicot-j.org/10.1051/sicotj/2021008.

Table 1s. International Survey on Surgeon Preferences in the Management of Stiffness following Total Knee Arthroplasty.

\section{Conflict of Interest}

All authors declare that they have no conflict of interest for the submitted work and have received no funding for this work. KHSK - Chair of SICOT Young Surgeons Committee.

VK - Educational consultant Smith \& Nephew \& Arthrex, Chair SICOT Education Academy, President Elect British Hip Society, Past Chair - NAHR UK.

\section{References}

1. Weinstein AM, Rome BN, Reichmann WM, et al. (2013) Estimating the burden of total knee replacement in the United States. J Bone Joint Surg Am 95, 385-392.

2. Mamarelis G, Sunil-Kumar KH, Khanduja V (2015) Timing of manipulation under anaesthesia for stiffness after total knee arthroplasty. Ann Transl Med 3, 316.
3. Nakano N, Shoman H, Olavarria F, et al. (2020) Why are patients dissatisfied following a total knee replacement? A systematic review. Int Orthop 44, 1971-2007.

4. Ritter MA, Harty LD, Davis KE, et al. (2003) Predicting range of motion after total knee arthroplasty. Clustering, log-linear regression, and regression tree analysis. J Bone Joint Surg Am $85,1278-1285$.

5. Witvrouw E, Bellemans J, Victor J (2013) Manipulation under anaesthesia versus low stretch device in poor range of motion after TKA. Knee Surg Sports Traumatol Arthrosc Off J ESSKA 21, 2751-2758.

6. Yercan HS, Sugun TS, Bussiere C, et al. (2006) Stiffness after total knee arthroplasty: Prevalence, management and outcomes. The Knee 13, 111-117.

7. Choi H-R, Siliski J, Malchau H, et al. (2014) How often is functional range of motion obtained by manipulation for stiff total knee arthroplasty? Int Orthop 38, 1641-1645.

8. Fox JL, Poss R (1981) The role of manipulation following total knee replacement. J Bone Joint Surg Am 63, 357-362.

9. Desai AS, Karmegam A, Dramis A, et al. (2014) Manipulation for stiffness following total knee arthroplasty: When and how often to do it? Eur J Orthop Surg Traumatol Orthop Traumatol 24, 1291-1295.

10. González Della Valle A, Leali A, Haas S (2007) Etiology and surgical interventions for stiff total knee replacements. HSS J Musculoskelet J Hosp Spec Surg 3, 182-189.

11. Kalson NS, Borthwick LA, Mann DA, et al. (2016) International consensus on the definition and classification of fibrosis of the knee joint. Bone Jt J 98-B, 1479-1488.

12. Ghani H, Maffulli N, Khanduja V (2012) Management of stiffness following total knee arthroplasty: A systematic review. The Knee 19, 751-759.

13. SICOT. The World Orthopaedic Organisation. http://www.sicot. org/about-sicot. Accessed 9 Feb 2021

14. Jones TL, Baxter MAJ, Khanduja V (2013) A quick guide to survey research. Ann R Coll Surg Engl 95, 5-7.

15. Namba RS, Inacio M (2007) Early and late manipulation improve flexion after total knee arthroplasty. J Arthroplasty 22, 58-61.

16. Issa K, Banerjee S, Kester MA, et al. (2014) The effect of timing of manipulation under anesthesia to improve range of motion and functional outcomes following total knee arthroplasty. J Bone Joint Surg Am 96, 1349-1357.

17. Yeoh D, Nicolaou N, Goddard R, et al. (2012) Manipulation under anaesthesia post total knee replacement: Long term follow up. The Knee 19, 329-331.

18. Kornuijt A, Das D, Sijbesma T, et al. (2018) Manipulation under anesthesia following total knee arthroplasty: A comprehensive review of literature. Musculoskelet Surg 102, 223-230.

19. Milne S, Brosseau L, Robinson V, et al. (2003) Continuous passive motion following total knee arthroplasty. Cochrane Database Syst Rev CD004260. https://doi.org/10.1002/14651858.CD004260.

20. Boese CK, Weis M, Phillips T, et al. (2014) The efficacy of continuous passive motion after total knee arthroplasty: A comparison of three protocols. J Arthroplasty 29, 1158-1162.

21. Vun SH, Shields DW, Sen A, et al. (2015) A national questionnaire survey on knee manipulation following total knee arthroplasty. J Orthop 12, 193-196. 